\title{
A Survey on Urban Traffic Optimisation for Sustainable and Resilient Transportation Network
}

\author{
Koh Song Sang, Bo Zhou, Po Yang, and Zaili Yang \\ Department of Computer Science, \\ Liverpool John Moores University, \\ Liverpool, L3 3AF, UK \\ S.S.Koh@2014.ljmu.ac.uk, \{B.Zhou,P.Yang,Z.Yang\}@ljmu.ac.uk
}

\begin{abstract}
Nowadays, sustainability and resilience have become a major consideration that cannot be neglected in urban development. People are starting to consider utilizing the urban infrastructure environment to maintain and improve the functionality and availability of the urban system when unexpected events take place. Traffic congestion is always a major issue in urban planning, especially when the vehicles in the roadway keep growing and the local authorities are lack of solutions to manage or distribute the traffics in the city. It has huge impact on urban sustainability and resilience such as overload of the city's infrastructure, and air pollution, etc. This paper presents a survey on the challenges of developing sustainable and resilient transportation networks and the current urban traffic optimisation methods, as a possible solution to address such challenges. It aims to describe and define the state of the art on the research on sustainable and resilient transportation networks in urban development and a taxonomy of different traffic optimisation methods used for avoiding traffic congestion and improve urban traffic management.
\end{abstract}

Index Terms - Urban Development, Traffic Congestion, Sustainable and Resilient Transport Network

\section{INTRODUCTION}

Over the last decade, the sustainability and resilience have become a major concern in urban development. Globally, more people live in urban areas than in rural areas, with total of 54 percent of the world's population now live in urban areas in 2014 , and by 2050,66 percent of the world's population are projected to be urban [1]. With the growing population, cities around the world are struggling to transform its infrastructure and make the changes in order to meet the daily basic needs economically and environmentally. These cause the potential damage of environment such as increased consumption of natural resources [2], excess of air pollution, noise and dust [3], and raising hazardous waste on the urban population [4]. Therefore, sustainable and resilient transport network development is playing an important role in urbanization.

In general, sustainable development aims at creating and maintaining our options for prosperous social and economic development [5]. It emphasizes an optimal balance between social needs, economy and environment. However, resilience development aims to provide the capacity to absorb the shock and maintain the availability of the function where unexpected event happen [6].

Sustainability and resilience are the key factors in urban transportation networks in order to make sure it will meet the basic needs of society and individuals without seriously damaging the environment and decreasing the safety level on the roadway. For instance, traffic congestion is always a major issue in urban planning, especially when the vehicles on the roadway keep growing and the urban infrastructure is not upgraded in equal speed to accommodate the growing number of vehicles. Besides, although many different methods about route optimization had been widely studied, most of them are only designed for individual user and lack of consideration from the urban wise in terms of sustainability and resilience. Furthermore, local authorities to date still lack efficient technical solutions in order to manage or distribute the traffics in the urban transportation network. To fulfill such research gaps, it is essential and significant to investigate the state of the art of research on sustainability and resilience of transport networks.

The rest of this paper is organized as below. The next section explains the definition of sustainable and resilient transportation networks. Section III discusses the main issues and challenges in urban transportation. Section IV reviews existing techniques used for optimizing urban traffic from the perspectives of sustainability and resilience. Lastly the paper concludes with a brief analysis of future directions in Section IV.

\section{DEFINITION OF SUSTAINABLE AND RESILIENT TRANSPORTATION NETWORK}

Transportation is linked to all aspects of urban life. Ensuring a comprehensive, accessible and integrated transportation system is essential to sustain social and economic development. Urban transportation plays the central role in urban life. If any reduction in performance happens it may compromise the city's operations across different areas and cause large and costly disruptions.

Essentially, there are no universally accepted definition of sustainable and resilient transportation [7]. Believingly it is an extended concept from sustainable and resilient development. In 1987, Brundtland Commission [8] started to define the meaning of the term "Sustainable Development" in the report "Our Common Future". It purposed Sustainable Development is to meet the needs of the present without compromising the 
ability of future generations to meet their own needs. Since this report became available, sustainable development raised the concern for the urbanization in terms of human wellbeing, environment and economic.

Transportation Research Board defined the Sustainable Development as, "Sustainability is about how environmental, economic, and social systems interact to their mutual advantage or disadvantage at various space-based scales of operation" in their report [9]. It purposed more mutual interactions between different aspects in sustainable development.

Furthermore, Eurofound [10] defined the Sustainable Development more precisely, "Sustainable development is the achievement of continued economic development without detriment to the environmental and natural resources." This actually describes the nature of sustainable development in modern urban planning for long term development, which means its ultimate goal is to work on the balance between the social needs and the impact on the environment.

Sustainable transportation is however an extension of the sustainable development in urban transportation. It aims to ensure that environment, social and economic considerations are factored into decisions affecting transportation activities [11]. Sustainable transportation systems offer greater flexibility, coordination and redundancy, allowing users to be distributed across a diverse portfolio of transport options and to transfer easily from one mode to another when required and associated with fuel consumption and air pollution control in order to minimize the impact of urban transportation.

On the other hand, resilient development could be traced back to 1973, Holling [12] conceived resilience as "a measure of the persistence of systems and of their ability to absorb change and disturbance and still maintain the same relationships between populations or state variables". In his definition resilience is a property of a system which is used to measure the availability level of the system to absorb unexpected changes or events.

Carpenter et al. [13] described resilience with three characteristics: (1) the amount of disturbance a system can absorb and still remain with the same state or domain of attraction; (2) the degree to which the system is capable of selforganization; and (3) the ability to build and increase the capacity for learning and adaptation. Based on these characteristics resilience is a configuration of the system to maintain its structure or function by absorbing the disturbance, and potentially re-organized the system to adapt to the disturbance.

Resilience is a crucial term in urbanization, at least as crucial as Sustainability is. This is because the urban system is composed by both human and nature, which both are not independent [14] but deeply linked together. Therefore it is important to align resilience with urban development.

By applying the definitions of resilience above, we could understand that a resilient transportation network describes a transportation system with the capacity to absorb disturbances and adapt to unexpected events in order to improve its safety and availability.
In summary, integrity of the above sustainability and resilience definitions enables to reveal that sustainable and resilient transportation network actually describes an intelligent urban transportation system which is able to use the urban infrastructure efficiently, economically and environmentally to meet the basic need of individuals in order to balance the impact on urban environment and natural resources, by minimizing the emission and waste and limiting the fuel consumption and by maximising the capacity to adapt to accidents or unexpected events and to improve the safety on the roadway [22] [23].

\section{KEY ISSUES AND CHALLENGES IN SUSTAINABLE AND RESILIENT TRANSPORTATION NETWORK}

When considering the future development of the urban areas, especially in cities with high population, it is always met with challenges dealing with sustainability and resilience. Sustainable and resilient transportation network emphasises an integrated system with social, environment and economic considerations. Therefore the range of issues are addressed widely to cover different categories. As shown in Figure 1, T.Litman and D.Burwell [7] listed a wide range of sustainability issues in social, environment and economic categories. However, they also acknowledged that although each issue fits into a specific category, practically they are potentially overlapped and dependant.

\begin{tabular}{lll}
\hline Economic & Social & Environmental \\
\hline Traffic congestion & Inequity of impacts & Air and water pollution \\
Mobility barriers & Mobility disadvantaged & Habitat loss \\
Accident damages & Human health impacts & Hydrologic impacts \\
Facility costs & Community interaction & DNRR \\
Consumer costs & Community liveability & \\
DNRR & Aesthetics & \\
\hline DNRR: Depletion of non-renewable resources.
\end{tabular}

Figure 1 Transportation Issue in Sustainability [7]

\section{A. Traffic Congestion}

Traffic congestion is the flip side of sustainable and resilient transport network. Nowadays traffic congestion is a major concern in many cities due to the growing number of vehicles and the development of the roadway is not expanding proportionally. Traffic congestion is the initial reason for causing other issues in urban transportation. For instance, heavy traffic congestion leads to a high probability of road accidents [15] and increased air pollution [16].

Generally, there are two types of traffic congestion, which are recurring traffic congestion and non-recurring traffic congestion [17]. Recurring traffic congestion is defined as a congestion that consistently happens at certain places during certain time. It usually is treated as a capacity problem and can be solved by increasing the roadway capacity. However, in sustainability perspective, increasing capacity is not the efficient way to reduce traffic congestion socially, environmentally and economically [18]. Therefore an alternative route path optimization is needed to distribute the traffic in order to reduce the traffic congestion.

Meanwhile, non-recurring traffic congestion occurs unpredictably. Its occurrence could be triggered by 
construction, inclement weather, accidents, and special events [18]. The detection of non-recurring traffic congestion is critically more difficult than the recurring traffic congestion because it requires real-time traffic information to predict the potential traffic congestion [17]. Improving roadway condition does not seem to be a good option because of its unpredictable nature. In this case, a good vehicle traffic system is needed to control the traffic in dealing with unexpected event.

A US Federal Highway Administration report [19] defined six sources of congestion as shown in Figure 2 which are (1) Bottleneck - vehicles stuck at narrow road; (2) Traffic Incidents - The delay of traffic because of vehicles crash or spoil; (3) Work Zones - road or building that beside main road are under construction or maintenance activities; (4) Bad Weather extreme weather such as heavy rain, snow, fog that can cause the congestion; (5) Poor Signal Timing - traffic light controller does not control the traffic signal efficiently with the time allocated for a signal doesn't match the traffic volume; (6) Special Event - Unexpected event that cause congestion, such as marathon, car racing.

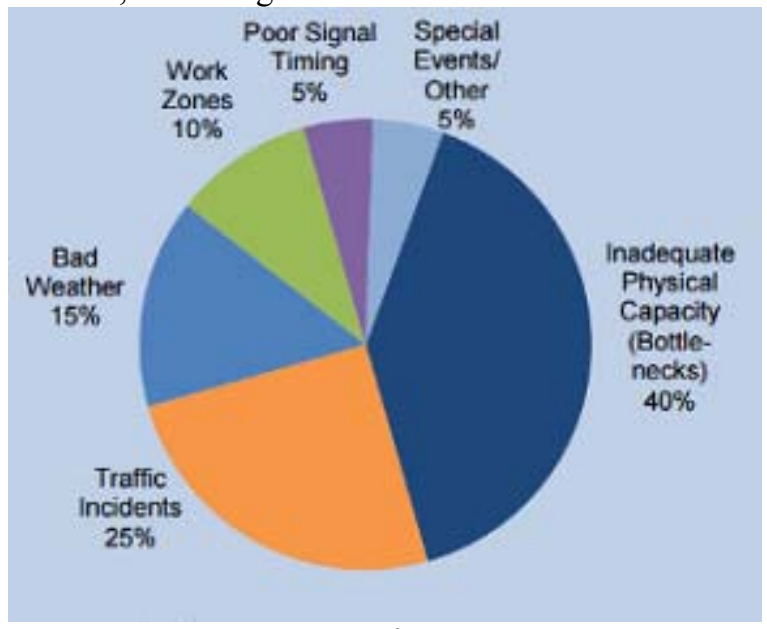

Figure 2 Cause of Congestion [19]

There are several challenges to prevent or reduce traffic congestions for sustainable and resilient transportation network.

- How to gather accurate and reliable real-time traffic information?

- How to intelligently manage and distribute traffics in urban transportation?

- How to prioritize the emergency vehicles to save lives and properties?

Designing an adaptive methodology to overcome these challenges can be a critical for future research towards sustainable and resilient transportation network.

\section{B. Centralized Vechicle traffic Management}

The difficulty of deploying centralized vehicle traffic management system is another issue for sustainable and resilient transport network. Urban transportation network is usually inefficient due to the bad decision making by individual driver. Therefore, a centralize vehicle traffic management system which is able to supervise the routing process for local authorities by monitoring the urban traffics, and react instantly against unexpected event in the traffic, is the key to build an intelligent urban transport network [20].

However, centralized vehicle traffic management system relies largely on the application of advanced technologies. Due to the high speed changes in traffic system and the wide distribution of vehicles on the roadway [21], centralized vehicle traffic management system requires sensing equipment deployed on each vehicle and advanced infrastructure on the roadway to adapt to the real-time traffic situation. Although some network technologies such as wireless sensor network is getting widely used, the lack of infrastructure in certain places may cause the delay in response from the central traffic control, when accident or unplanned event takes place. It is especially more evident for those developing countries with high population density.

Therefore, the main challenge of designing a centralized traffic management system relies on whether the infrastructure of the urban environment could support a reliable communication between vehicles and roadside infrastructure in order to provide rapid information sharing.

\section{Vehicle Routing Problem}

Vehicle routing problem is another major challenge in sustainable and resilient transport network. Route optimization is a general method for resolving Vehicle Routing Problem (VRP) which is described as the problem of designing optimal delivery or collection routes from one or several depots to a number of geographically scattered cities or customers, subject to side constraints [30].

Dijkstra [24] proposed a static algorithm to find the shortest path without considering any external factor such as congestion, accident, or average vehicle speed. However, this method is not practical enough as the traffic of transport network is changing with time and specific constraints. Therefore vehicle routing optimization should always optimize the path continuously with the real-time information and adapt to the latest circumstance.

Besides, most of the route optimization methodologies are designed to optimize individual route path instead of maximizing the capacity of urban road network in order to reduce the traffic congestion. It is challenging to develop an algorithm which is able to make reaction and adaption to the complex, rapidly changing urban transport network while managing the urban traffic.

\section{URBAN TRAFFIC OPTIMIZATION}

There are several attempts and studies to resolve a traffic congestion problem for sustainable and resilient transportation network. In this paper we focus on two main categories which are vehicle routing optimization by using Ant-Colony algorithm and vehicle traffic management system by using wireless sensor network. This section evaluates some of the researches, and analyses both the strengths and weaknesses of the proposed solution.

\section{A. Ant-Colony Algorithm}

Ant-colony algorithm was first proposed by Dorigo et al. in paper [25] which was inspired by the natural behaviour 
performed by ants in finding food resources. In this natural phenomenon, ants leave pheromone trails to allow other ants to track the path and eventually find the food resources. Previous experiments have proven that ants are able to find the shortest route between two individual sections. Therefore ant-colony algorithm is widely applied to solve vehicle routing problem, although some modifications have been applied depending on different circumstances.

Nahar and Hashim [26] proposed a traffic congestion control method based on different preferences to create an optimal traffic system. Figure 3 shows the method used in determining the optimum path. These preferences allow the algorithm to reduce average travelling time by adjusting the ant colony variables. Their results show that the number of ants is directly correlated with the algorithm performance. Therefore, this method does not perform well when there is only a small number of agents in the network.

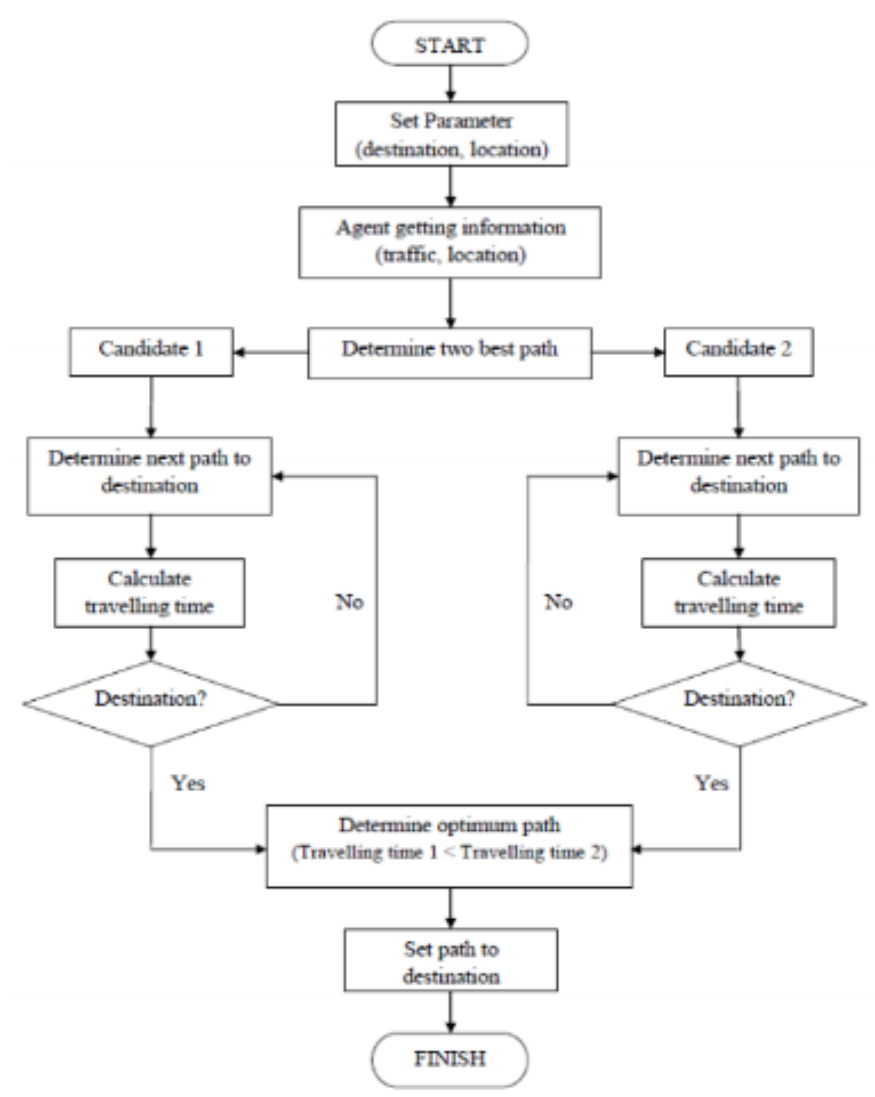

Figure 3 Flowchar for determining the optimum path using the Ant-Colony Optimization algorithm [26]

Kammoun et al. [27] however proposed an adaptive vehicle guidance system which is able to search for the best path in a smarter way by using real-time changes in the network. In order to achieve dynamic traffic control and improve driver's request management, this method used three types of agents, which are:

- Intelligent vehicle-ant agent (IVAA): standing for a vehicle and encapsulates a deliberative module for the selection of the best itinerary alternative.
- Road Supervisor Agent (RSA): Representing a software agent implanted in the server.

- $\quad$ City Agent (CA): Representing a software agent to manage the road network in the city in order to obtain a better exploitation of the network.

Besides, a multi-agent evacuation model was introduced by Zong et al. [28] to minimise the total evacuation time for vehicles and balance the traffic load. Figure 4 shows the route optimization process by using two ant colonies. Ants belong to one colony find their routes with the same properties. During the process of searching for routes, the two ant colonies will interact and share information with each other. Experiments have shown that Multi-Ant colony system is more effective than a single agent system.

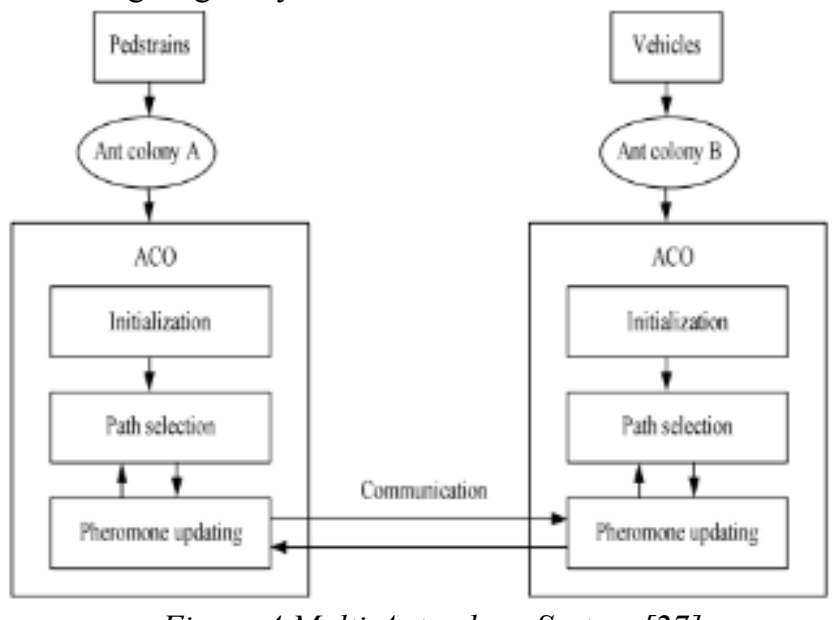

Figure 4 Multi-Ant colony System [27]

Cong et al. [29] developed a model to optimise dynamic traffic routing by using a two-steps approach: network pruning and network flow optimisation. In the network-pruning phase, ant pheromone is removed after the best route is found by the agents to increase exploration rate. In the flow optimisation phase, which is based on Ant-Colony Optimization with the stench pheromone and colored pheromone, the agents correspond to the links selected in the network-pruning phase only. Moreover, this two-steps approach effectively reduces the computing burden when addressing complex and dynamic traffic control problems.

Kponyo et al. [15] proposed a distributed intelligent traffic system which uses vehicle average speed as a parameter to determine the traffic condition. This system guides vehicles to paths with low traffic. Therefore, this system selects the best path more efficiently comparing with scenarios where the agents select their path randomly.

\section{B. Wireless Sensor Network}

Nowadays, the wireless sensor network is getting more widely applied and it potentially provides a very solid technical foundation for vehicle traffic management system applications. For instance, the mobility of sensor nodes enables the Vehicle to Vehicle (V2V), Vehicle to Roadside (V2R) or Vehicle to Infrastructure (V2I) communication, which means the data sharing and collection are more reliable and fast between 
vehicles, roadside and infrastructures. By accessing the realtime traffic data, the vehicle traffic management system controller could response instantly to control the urban traffic flow [17].

Chao et al. [31] proposed an intelligent traffic management system which focuses on the use of radio frequency identification (RFID) as a form of traffic flow detection. The proposed intelligent traffic light control system (ITLCS) uses an RFID system to detect the number of seconds spent by vehicles on main roads and on side roads passing through detectors during a green light period and then transmits the detected data to the program system on chip ( $\mathrm{PSoC}$ ) microcontroller. They used Zig Bee wireless modules (a wireless network protocol for high-level communication used) to send real time data regarding weather conditions, air quality, and the detected vehicle registration information to the regional control centre. The proposed system is able to perform remote transmission and reduce the risk of accident.

Bruno et al. [32] proposed two efficient data collection algorithms which are GREEDY and Probabilistic Data Collection (PDC) for vehicular multimedia sensor networks. The project simulated the proposed algorithm using a VANETMobiSim and NS-2 simulator. The simulation results show that the GREEDY solution can achieve a more uniform coverage and consume less network bandwidth.

Choi et al. [33] designed an optimal routing policy for the vehicular sensor network (VSN). This project developed a delay-optimal VSN routing algorithm by capturing three key features in urban VSNs: (i) vehicle traffic statistics, (ii) any cast routing and (iii) known future trajectories of vehicles such as bus. Figure 3 shows the roadmap and its correspondingly directed graph G. In Figure 5(a), two Wi-Fi Access points (AP) are placed at the intersections $\mathrm{i}, \mathrm{i} 9$ and the path of bus $\mathrm{A}$ is the sequence of intersections, i1, i2, i5, i8 and i9. However, the Figure 5(b) represents a network that can be potentially used for delivering data packets, and the existence of data links in the network is highly uncertain. They conducted simulations on a GloMoSim simulator and compared the performance of Optimal VSN Data Forwarding with the Vehicle Assisted Data Delivery (VADD) algorithm and Trajectory Based Data (TBD) forwarding scheme. The simulation results show that the OVDF outperforms other algorithms.

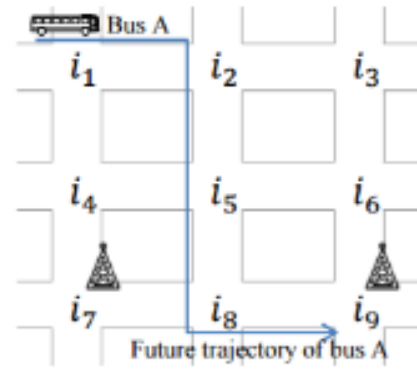

(a) Roadmap

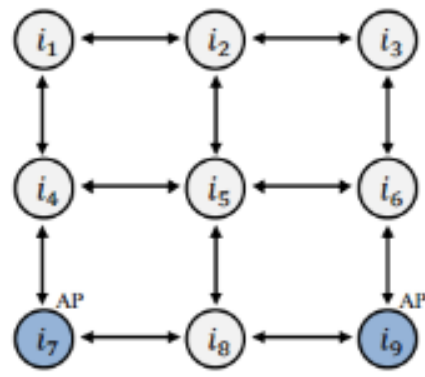

(b) Road network graph $G$
Figure 5 Roadmap and its corresponding road network graph [33].

Bharadwaj et al. [34] proposed an Efficient Dynamic Traffic Control System (EDTCS) to reduce vehicle traveling time and set the highest priority for emergency vehicles at intersections. EDTCS is composed by Traffic Control Unit (TCU), Traffic Monitor Unit (TMU) and Road Side Unit (RSU). Figure 6 shows the process at the intersection. All the vehicles in the sensor area are counted and emergency vehicles are identified by RFID tags. Those emergency vehicles are able to communicate with the RSU via RFID tags. If the RFID tag is positive, RSU will increase the emergency vehicle's number by one. Then the centralized traffic server will collect the number of both normal and emergency vehicles and switch the traffic signal to green for about 30 seconds for the side of emergency vehicles to go through the intersection.

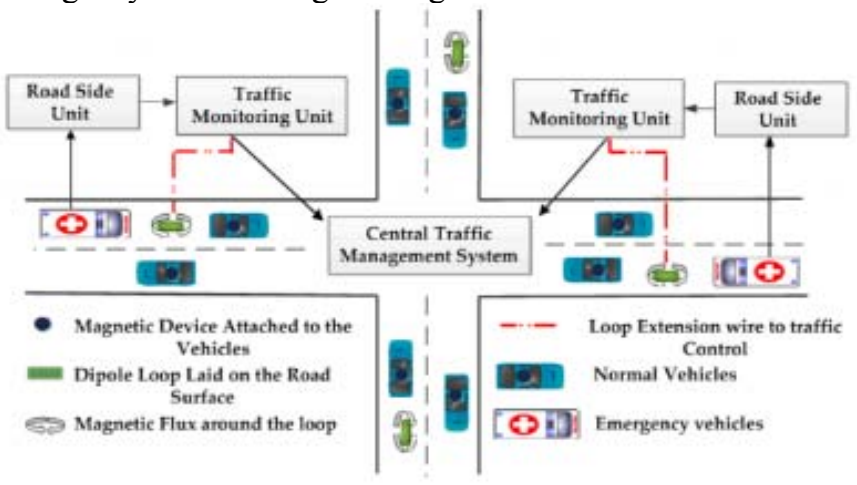

Figure 6 Layout architecture for efficient dynamic traffic control system [34]

\section{CONCLUSIONS AND FUTURE WORK}

Sustainability and resilience of urban transportation plays important role in urbanization which is responsible for connecting different aspects and properties in urban environment. Ensuring a comprehensive, accessible and integrated transportation system is essential in urban development. A sustainable and resilient transportation network aims to meet the individual transportation needs without compromising the wide interests and provide an adaptive capacity to absorb the unexpected events to maintain the function of transportation network.

Developing towards sustainable and resilient transportation network is therefore of significance while being of huge challenges, wanting solutions to be found. . First of all, traffic congestion is a raising concern for urban development. Especially the non-recurring traffic congestion which is hard to be predicted. Secondly, the limitation of existing infrastructure in many cities cannot provide reliable network to support rapid information sharing for centralized traffic management system. Lastly, most of the path selection methods are designed for individual usage. There is a need for a design of route optimization algorithm that based on urban prospective in consideration of both sustainability and resilience.

In this paper, we conducted a brief survey on the issues of sustainable and resilient transportation network and existing solutions, with a particular focus on Ant-Colony Algorithm for vehicle routing problem and wireless sensor networks for traffic management system. In the future work it looks promising to evaluate a new route optimization method which will be able to 
work with wireless sensor network to provide decision support service to distribute the urban traffics for local authorities.

\section{REFERENCE}

[1] World Urbanization Prospects 2014, United Nations, 2014. [2] S. Mukherji, Migration and Urban Decay. Asian Experiences, Rawat Publication, Bangalore. 2006.

[3] S.Uttara, N. Bhuvandas, V. Aggarwal. Impacts of Urbanization on Environment. International Journal of Research in Engineering \& Applied Sciencec. 2014.

[4] Muthukumaran and Ambujam,N.K. Wastewater Treatment and New York, Department of Economic and Social Affairs Population Division. 2003.

[5] Folke, C., S. Carpenter, T. Elmqvist, L. Gunderson, C.S. Holling and B. Walker. Resilience and Sustainable Development: Building Adaptive Capacity in a World of Transformations. Ambio 31 (5), pp.437-440. 2002

[6] Gunderson LH and Pritchard L. editors. Resilience and the Behavior of Large-Scale Ecosystems. Island Press. 2002

[7] Todd Litman, David Burwell. Issues in sustainable transportation. Int. J. Global Environmental Issues. 2006.

[8] World Commission on Environment and Development (Brundtland Commission). Our Common Future, Oxford University Press, Oxford, UK. 1987.

[9] Transportation Research Board. Toward a Sustainable Future, Special Report 251, Washington DC. 1997.

[10] Sustainable Development. European Foundation for the Improvement of Living and Working Conditions. 2004.

[11] Moving on Sustainable Transportation (MOST) Transport Canada. 1999.

[12] Holling, C.S. Resilience and stability of ecological systems. Annual Review of Ecology and Systematics 4, pp.1-23. 1973.

[13] Carpenter, S.R., B.H. Walker, J.M. Anderies and N. Abel. From metaphor to measurement: resilience of what to what? Ecosystems 4, pp.765-781. 2001.

[14] Walker, B. and D. Salt. Resilience thinking: sustaining ecosystems and people in a changing world. Washington: Island Press. 2006.

[15] Kponyo, J.J., Kuang, Y. and Li, Z. 'Real time status collection and dynamic vehicular traffic control using ant colony optimization. International Conference on Computational Problem-Solving (ICCP), pp.69-72.2012.

[16] Kai Zhang, Stuart Batterman. Air pollution and health risks due to vehicle traffic. Science of the Total Environment 450451 (2013) 307-316. January 2013.

[17] Kapileswar Nellore and Gerhard P. Hancke. A Survey on Urban Traffic Management System Using Wireless Sensor Networks. January 2016.

[18] Jessica McGroarty. Recurring and Non-Recurring Congestion: Causes, Impacts, and Solutions. January 2010.

[19] Status of the Nation's Highways, Bridges, and Transit: Conditions and Performance; US Federal Highway Administration Report. US Department of Transportation: Washington, DC, USA, 2013.

[20] Schmitt, E. J., \& Jula, H. Vehicle Route Guidance Systems: Classification and Comparison. Proceedings of the IEEE Intelligent Transport Systems Conference, 242-247. 2006.
[21] Sj obergBilstrup, K. Predictable and Scalable Medium Access Control for Vehicular ad Hoc Networks, PhD thesis, Halmstad University. 2009.

[22] Secretary of State for Transport. Towards a Sustainable Transport System - Supporting Economic Growth in a Low Carbon World. October 2007.

[23] Hoogma, R., R. Kemp, J. Schot, and B. Truffer, Experimenting for Sustainable Transport: The Approach of Strategic Niche Management. Routledge, London, New York. 2002

[24] E. Dijkstra: ' A Note on Two Problems in Connexion with Graphs', Numerische Mathematik, Vol. 1, pp. 269-271. 1959.

[25] Dorigo, M., Maniezzo, V., \& Colorni, A. Ant System: Optimization by a colony of cooperating agents. IEEE Transactions on Systems, Man, and Cybernetics-Part B, 26(1), 29-41. 1996

[26] Nahar, S. A.A. and Hashim, F.H. 'Modelling and analysis of an efficient traffic network using ant colony optimization algorithm', in Third International Conference on Computational Intelligence, Communication Systems and Networks (CICSyN), pp.32-36, IEEE. 2011

[27] Kammoun, H.M., Kallel, I., Alimi, A.M. and Casillas, J. 'An adaptive vehicle guidance system instigated from ant colony behavior', IEEE International Conference on Systems Man and Cybernetics (SMC), pp.2948-2955, IEEE.

[28] Zong, X., Xiong, S., Fang, Z. and Li, Q. (2010) 'Multi-ant colony system for evacuation routing problem with mixed traffic flow', in IEEE Congress on Evolutionary Computation (CEC), pp.1-6, IEEE. 2010.

[29] Cong, Z., DeSchutter, B. and Babuska, R. 'A new ant colony routing approach with a trade-off between system and user optimum', in 14th International IEEE Conference on Intelligent Transportation Systems (ITSC), pp.1369-1374, IEEE. 2011.

[30] Gilbert Laporte. The Vehicle Routing Problem: An Overview of Exact and Approximate Algorithms. 1991

[31] Chao, K.H.; Chen, P. An intelligent traffic flow control system based on radio frequency identification and wireless sensor networks. Int. J. Distrib. Sens. Netw. 2014.

[32] R.Bruno, M.Nurchis. Robust and efficient data collection schemes for vehicular multimedia sensor networks. In Proceedings of the IEEE 14th International Symposium and Workshops on World of Wireless, Mobile and Multimedia Networks (WoWMoM), Madrid, Spain. 2013

[33] O. Choi, S. Kim, J. Jeong. W. H. Lee, S. Chong. Delayoptimal data forwarding in vehicular sensor networks. In Proceedings of the IEEE 11th International Symposium on Modeling and Optimization in Mobile, Ad Hoc and Wireless Networks (WiOpt), Tsukuba Science City, Japan, 13-17 May 2013; pp. 532-539. 2013.

[34] R. Bharadwaj, J. Deepak, M. Baranitharam, V. Vaidehi. Efficient dynamic traffic control system using wireless sensor networks. In Proceedings of the IEEE International Conference on Recent Trends in Information Technology (ICRTIT), Chrompet, Chennai, India, 25-27 July 2013; pp. 668-673. 2013. 